\title{
Establishment of an immortalized cell line derived from the prairie vole via lentivirus-mediated transduction of mutant cyclin-dependent kinase 4, cyclin $\mathrm{D}$, and telomerase reverse transcriptase
}

\author{
Masafumi KATAYAMA ${ }^{1,2)}$, Tohru KIYONO ${ }^{3)}$, Kengo HORIE ${ }^{1)}$, Takashi HIRAYAMA ${ }^{1,4)}$, \\ Takahiro EITSUKA ${ }^{5)}$, Kengo KURODA ${ }^{1,2)}$, Kenichiro DONAI ${ }^{1,2)}$, Shizu HIDEMA ${ }^{1)}$, \\ Katsuhiko NISHIMORI ${ }^{1)}$, and Tomokazu FUKUDA ${ }^{1)}$ \\ ${ }^{1)}$ Graduate School of Agricultural Science, Tohoku University, 1-1 Tsutsumidori-amamiyamachi, Aoba-ku, Sendai \\ 981-8555, Japan \\ 2) Japan Society for the Promotion of Science, 5-3-1, Kojimachi, Chiyoda-ku, Tokyo 102-0083, Japan \\ 3) Division of Carcinogenesis and Cancer Prevention, National Cancer Center Research Institute, Tsukiji, Chuo-ku, \\ Tokyo 104-0045, Japan \\ 4) Department of Obstetrics and Gynecology, Juntendo University, 2-1-1, Hongo, Bunkyo-ku, Tokyo 113-8421, Japan \\ 5) Faculty of Applied Life Sciences, Niigata University of Pharmacy and Applied Life Sciences, 265-1, Higashijima, \\ Akiha-ku, Niigata 956-8603, Japan
}

\begin{abstract}
The prairie vole (Microtus ochrogaster) shows social behaviors such as monogamy and parenting of infants with pair bonding. These social behaviors are specific to the prairie vole and have not been observed in other types of voles, such as mountain voles. Although the prairie vole has several unique characteristics, an in vitro cell culture system has not been established for this species. Furthermore, establishment of cultured cells derived from the prairie vole may be beneficial based on the three Rs (i.e., Replacement, Reduction, and Refinement) concept. Therefore, in this study, we attempted to establish an immortalized cell line derived from the prairie vole. Our previous research has shown that transduction with mutant forms of cyclin-dependent kinase 4 (CDK4), cyclin D, and telomerase reverse transcriptase (TERT) could efficiently immortalize cells from multiple species, including humans, cattle, pigs, and monkeys. Here, we introduced these three genes into prairie vole-derived muscle fibroblasts. The expression of mutant CDK4 and cyclin D proteins was confirmed by western blotting, and telomerase activity was detected in immortalized vole muscle-derived fibroblasts (VMF-K4DT cells or VMFs) by stretch PCR. Population doubling analysis showed that the introduction of mutant CDK4, cyclin D, and TERT extended the lifespan of VMFs. To the best of our knowledge, this is the first report describing the establishment of an immortalized cell line derived from the prairie vole through the expression of mutant CDK4, cyclin D, and human TERT.
\end{abstract}

Key words: cellular senescence, immortalized cell, prairie vole, rodents

(Received 17 June 2015 / Accepted 31 August 2015 / Published online in J-STAGE 23 October 2015)

Address corresponding: T. Fukuda, Graduate School of Agricultural Science, Tohoku University, 1-1 Tsutsumidori-amamiyamachi, Aoba-ku, Sendai 981-8555, Japan

T. Kiyono, Division of Carcinogenesis and Cancer Prevention, National Cancer Center Research Institute, Tsukiji, Chuo-ku, Tokyo 104-0045, Japan

(C)2016 Japanese Association for Laboratory Animal Science 


\section{Introduction}

The prairie vole (Microtus ochrogaster) is an experimental rodent exhibiting unique social behavior. Voles form socially monogamous pair bonds [1], which may be explained by intracranial signals, such as the vasopressin and oxytocin hormone signals $[6,21]$. Interestingly, this social behavior has never been observed in other types of voles, such as mountain voles. Although the social behaviors of prairie voles are regulated by a high-grade neural mechanism, the detailed mechanisms occurring in the cerebral tissue are largely unknown.

Therefore, it is necessary to study the regulatory mechanisms that control these behaviors. Such experiments examining the neural mechanisms of social behavior require prairie voles to be subjected to painful experiments, such as neurosurgery. To reduce animal suffering, the concept of the three Rs (i.e., Replacement, Reduction, and Refinement) should be followed. Accordingly, establishment of an immortalized cell line derived from prairie voles would facilitate the study of these mechanisms in vitro, replacing animal studies with cellular analyses. Although primary cells from prairie vole tissues can be studied, these cells have not been shown to have the capacity for continued cell proliferation due to the occurrence of cellular senescence.

The characteristics of cellular senescence were first described by Heyflick $[9,10]$. The expression of oncogenic proteins, such as SV40T or human papillomavirusderived E6/E7 proteins, has been reported to efficiently immortalize primary cells [20]. However, the expression of these oncogenes sometimes changes the original features of cells, a process characterized as "oncogenic transformation". In 2011, Shiomi et al. reported that human fibroblasts can be immortalized using mutants of cyclin-dependent kinase (CDK) 4, cyclin D, and telomerase reverse transcriptase (TERT) with an intact karyotype [18]. Furthermore, such immortalized cells show differentiation capacity, suggesting that the expression of mutant CDK4, cyclin D, and TERT facilitates cellular immortalization while maintaining the original phenotype of the primary cells.

In senescent cells, p16 protein expression is increased. The p16 protein binds to CDK4 and inhibits the enzymatic activity of the CDK4/cyclin D complex, thereby reducing the turnover rate of the cell cycle. Therefore, it is necessary to characterize the level of conservation for these cell cycle-related genes. Among multiple spe- cies, the primary amino acid sequences of these proteins are well conserved, showing around $90 \%$ identity. Based on this finding, we showed that expression of mutant CDK4, cyclin D, and TERT could facilitate the establishment of immortalized cells from multiple species, including cattle, swine, and monkeys $[5,12]$. In this study, we attempted to establish immortalized cells derived from the prairie vole by expressing mutant CDK4, cyclin D, and TERT.

\section{Material and Methods}

Preparation of vole fibroblasts and culture conditions

The handling and maintenance of prairie voles were approved by the animal committee of Tohoku University. A small $(1 \times 1 \mathrm{~cm})$ piece of skeletal muscle tissue was dissected from adult voles (approximately 1 year of age). The muscle tissue was placed on a collagen-treated plastic dish. Both the primary and immortalized cells were cultured in Dulbecco's modified Eagle medium (DMEM; Nacalai Tesque, Kyoto, Japan) containing 10\% fetal bovine serum (Corning, Corning, NY, USA), and $1 \%$ Antibiotic-Antimycotic Mixed Stock Solution (Nacalai Tesque). All cells were maintained at $37^{\circ} \mathrm{C}$ with $5 \% \mathrm{CO}_{2}$.

\section{Establishment of an immortalized cell line derived from the prairie vole}

In order to immortalize vole-derived fibroblasts, CSIICMV-hCDK4R24C, CSII-CMV-cyclinD1, and CSIICMV-TERT were used to produce recombinant lentiviruses by transient expression in $293 \mathrm{~T}$ cells. A detailed description of this method of virus production was provided in our previous manuscripts [5, 17]. The production of recombinant lentiviruses using vesicular stomatitis virus $G$ glycoprotein was described in a previous study by Miyoshi [13].

\section{Population doubling (PD) assay}

Primary and immortalized vole cells were seeded in six-well plates at a density of $5 \times 10^{4}$ cells per well. When the cells reached confluence, both the primary and immortalized cells were trypsinized, and the number of cells per dish was counted using a cell counter. PD was used as the measure the cell growth rate and was calculated using the formula $\mathrm{PD}=1 / 2 \log _{2}$ (A / B), where $\mathrm{A}$ is the number of harvested cells and $B$ is the number of plated cells $[8,16]$. We analyzed the PD in triplicate and 
calculated the average and standard deviation (SD). Therefore, the PD value represents the number of cell divisions.

\section{Cell cycle analysis}

Cell cycle analyses of both the parental primary fibroblasts and the immortalized cells were performed using a Muse Cell Cycle Assay Kit (Merck Millipore Corporation, Darmstadt, Germany) and Muse Cell Analyzer (Merck Millipore Corporation) according to the manufacturer's protocols.

\section{Senescence-associated $\beta$-galactosidase staining}

Senescence-associated $\beta$-galactosidase staining was conducted to detect cellular senescence [4] using a Senescence Detection Kit (BioVision, Inc., Milpitas, CA, USA) according to the manufacturer's protocol.

\section{Western blotting}

We performed western blotting to detect the expression of proteins encoded by the introduced genes (i.e., CDK4 and cyclin D). Mouse anti-human cyclin D antibodies (1:4,000 dilution; BD Biosciences, Franklin Lakes, NJ, USA), mouse anti-human CDK4 antibodies (1:8,000 dilution; MBL, Nagoya, Japan), and mouse anti- $\alpha$-tubulin antibodies (1:1,000 dilution; Santa Cruz Biotechnology, Dallas, TX, USA) were used as the primary antibodies. Detailed methods for protein extraction, sodium dodecyl sulfate polyacrylamide gel electrophoresis (SDS-PAGE), and application of secondary antibodies have been previously published [5].

\section{Detection of telomerase activity}

Telomerase activity was determined by stretch PCR using a TeloChaser Kit (Toyobo, Osaka, Japan), which included a 65 -base pair internal control to facilitate quantification of activity. Briefly, $1 \times 10^{6}$ cells were homogenized in a lysis solution (Toyobo). The cell lysate (20 $\mu \mathrm{l}$; equivalent to $1.0 \times 10^{5}$ cells) was subjected to stretch PCR. Amplification was performed using the following thermal cycling conditions: $2.5 \mathrm{~min}$ of denaturation at $95^{\circ} \mathrm{C}$, followed by $30 \mathrm{~s}$ of denaturation at $95^{\circ} \mathrm{C}$, $30 \mathrm{~s}$ of annealing at $58^{\circ} \mathrm{C}$, and $45 \mathrm{~s}$ of extension at $72^{\circ} \mathrm{C}$. The PCR products were separated using 10\% acrylamide gel electrophoresis and stained with GelRed (Biotium, Inc., Hayward, CA, USA). The results of the staining were visualized using a ChemiDoc MP system (Bio-Rad, Hercules, CA, USA).

\section{Karyotype analysis}

We analyzed two immortalized vole cell lines. Cells were treated with colcemid prior to collection. After fixation, the cells were stained using G-band analysis methods. The chromosome number was determined from 50 mitotic cells. Detailed chromosome conditions with G-banding patterns were determined from 20 mitotic samples (metaphase).

\section{Statistical analysis}

The statistical significance of SA- $\beta$-Gal staining was evaluated using the Mann-Whitney U test. The level of significance was $5 \%$.

\section{Results}

Immortalization of vole fibroblasts by transduction with mutant CDK4, cyclin D, and TERT

First, we attempted to immortalize vole fibroblasts obtained from muscle tissue (Fig. 1A) by expression of mutant CDK4, cyclin D, and TERT (Fig. 1B). The expression levels of these genes did not induce any changes in cell morphology (Figs. 1A and B). We successfully obtained two cell lines (VMF-K4DT No. 1 and No. 2). VMF-K4DT No. 1 cells were established from a male vole, whereas VMF-K4DT No. 2 cells were established from a female vole.

Next, we analyzed the VMF-K4DT No. 1 cell line using population doubling level (PDL) assays. While the proliferation of primary fibroblasts was arrested, VMFK4DT No. 1 cells maintained stable cell proliferation (Fig. 1C). These data suggested that vole fibroblasts could be efficiently immortalized by the introduction of mutant CDK4, cyclin D, and TERT. In sequential passaging, VMF-K4DT No. 2 cells did not show any signs of cellular senescence up to passage 5 (data not shown).

\section{Detection of CDK4, cyclin D, and TERT}

As shown in Fig. 1, vole fibroblasts could be immortalized by introduction of mutant CDK4, cyclin D, and TERT. First, we evaluated the protein expression levels of mutant CDK4, cyclin D, and TERT, which were introduced by lentiviral infection. CDK4 and cyclin D were detected in VMF-K4DT No. 1 cells at the expected molecular weights; however, the expression of these proteins was not detected in primary cells (Fig. 2A). Thus, we concluded that these proteins were expressed as a result of lentiviral infection. Furthermore, we analyzed 

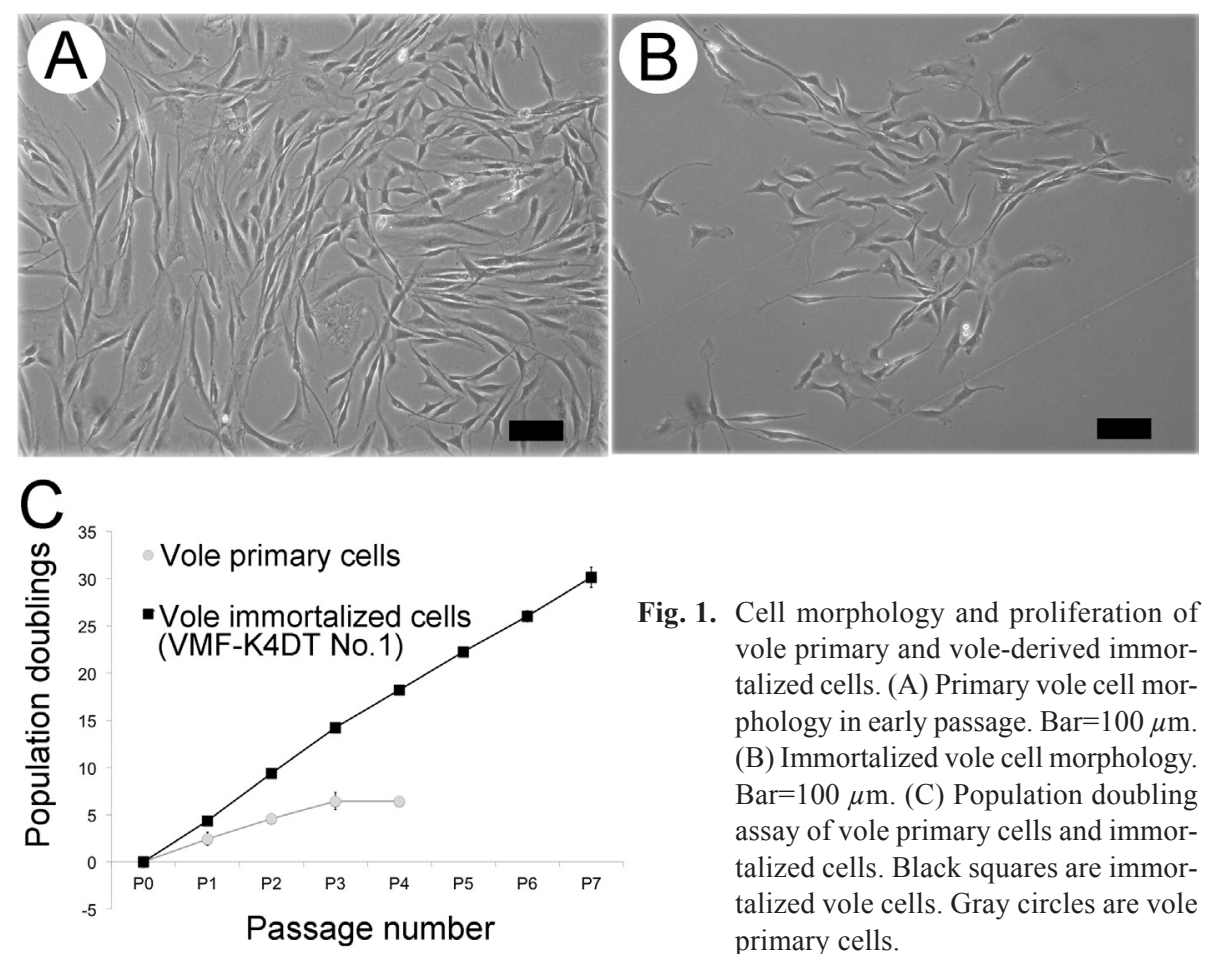

Fig. 1. Cell morphology and proliferation of vole primary and vole-derived immortalized cells. (A) Primary vole cell morphology in early passage. Bar $=100 \mu \mathrm{m}$. (B) Immortalized vole cell morphology. Bar $=100 \mu \mathrm{m}$. (C) Population doubling assay of vole primary cells and immortalized cells. Black squares are immortalized vole cells. Gray circles are vole primary cells.

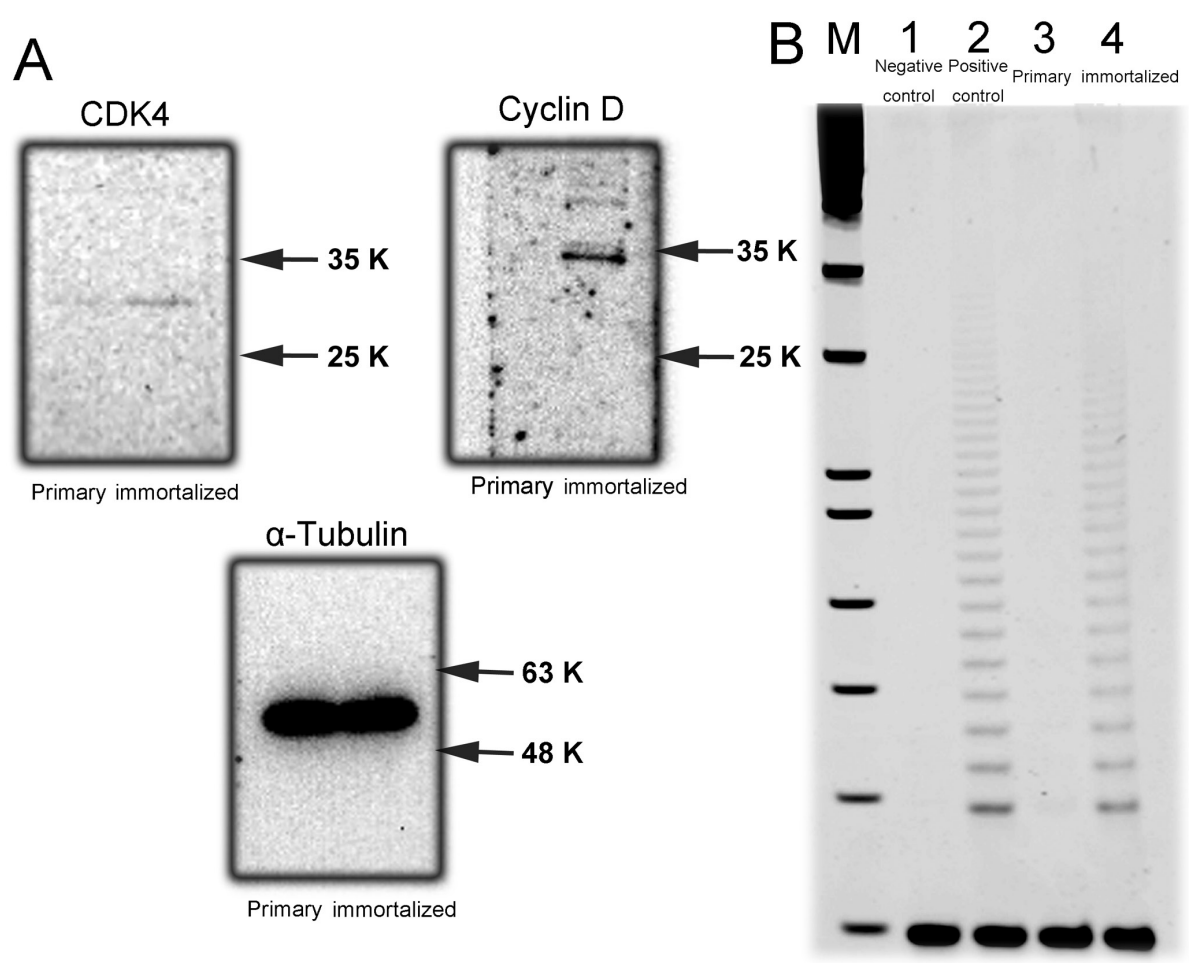

Fig. 2. Detection of the expression of mutant CDK4, cyclin D, and TERT in immortalized vole cells. (A) Detection via western blotting (CDK4 and cyclinD). Alpha-tubulin was used as a loading control. Vole primary cells are shown in the left lane, and immortalized vole cells are shown in the right lane. (B) Detection of telomerase activity. M, ladder (FX174/Hinf I digest); 1, negative control; 2, positive control (HeLa cells); 3, primary vole cell; and 4, immortalized vole cell. 

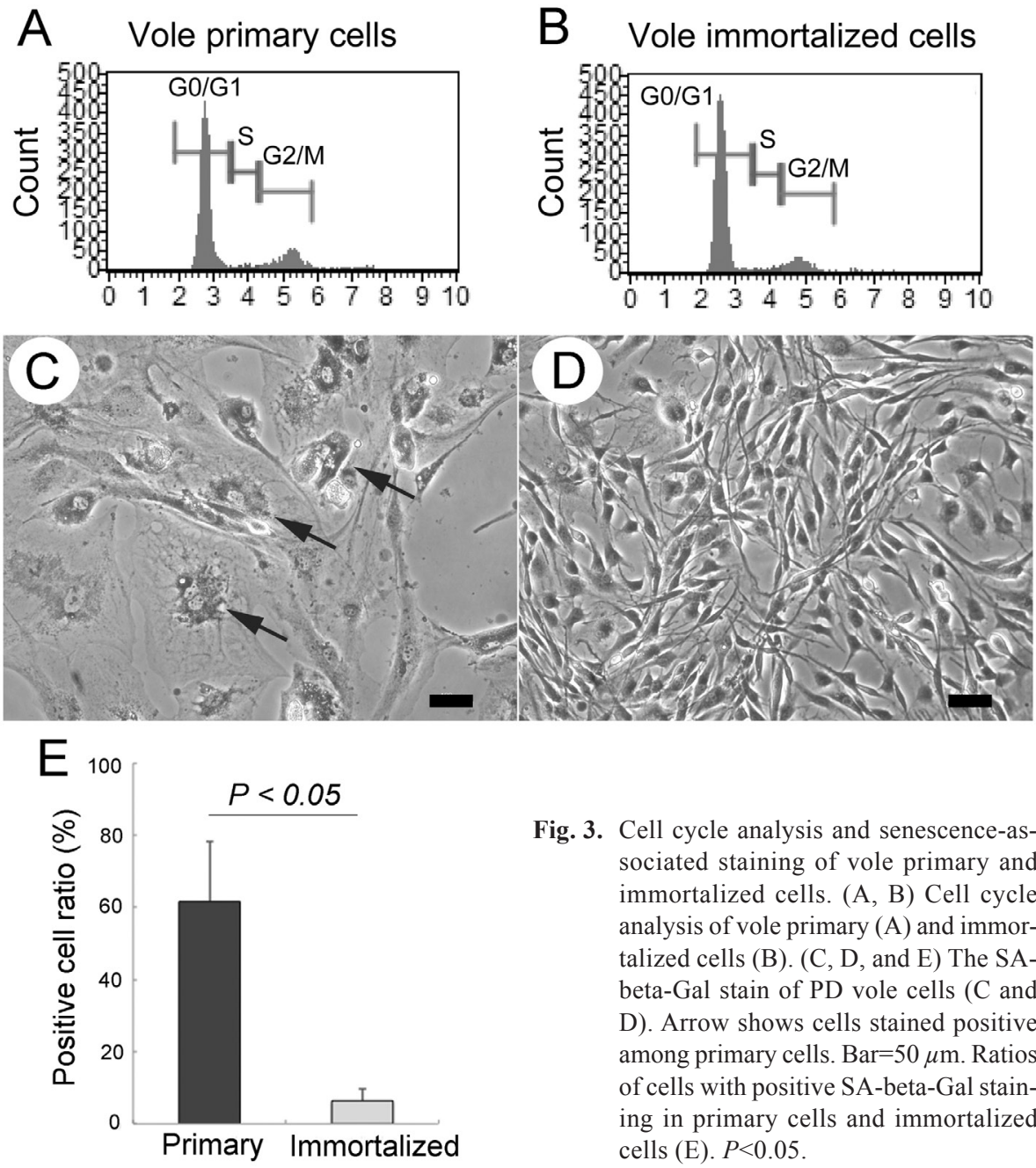

Fig. 3. Cell cycle analysis and senescence-associated staining of vole primary and immortalized cells. (A, B) Cell cycle analysis of vole primary (A) and immortalized cells (B). (C, D, and E) The SAbeta-Gal stain of PD vole cells (C and D). Arrow shows cells stained positive among primary cells. Bar $=50 \mu \mathrm{m}$. Ratios of cells with positive SA-beta-Gal staining in primary cells and immortalized cells (E). $P<0.05$.

telomerase activity in order to confirm that TERT was expressed in vole fibroblasts. Telomerase elongates the telomere sequence, which is a repetitive sequence at the ends of chromosomes. The telomerase complex consists of two components: telomerase RNA component (TERC) and TERT. In stretch PCR assays, telomerase activity was observed in TERT-induced cells (Fig. 2B), demonstrating that telomerase activity could be detected from transfection with TERT and from endogenous vole TERC in vole fibroblasts.

Cell cycle analysis and lack of cellular senescence in VMF-K4DT No. 1 cells

Next, we compared the cell cycle distributions of vole primary cells and VMF-K4DT cells. There were no significant differences in the percentages of cells within the $\mathrm{G}_{0} / \mathrm{G}_{1}, \mathrm{~S}$, and $\mathrm{G}_{2} / \mathrm{M}$ phases for primary cells and VMF-
K4DT No. 1 cells (Figs. 3A, B and Table 1), suggesting that cell cycle turnover was maintained in VMF-K4DT No. 1 cells. Subsequently, we used SA- $\beta$-Gal staining in order to detect cellular senescence in later stages with PDL assays. SA- $\beta$-Gal staining is a common method for detecting cellular senescence [4]. The positive cell ratio of SA- $\beta$-Gal staining in primary cells was significantly increased compared with that in VMF-K4DT No. 1 cells (Figs. 3C-E). These results suggested that VMF-K4DT No. 1 cells were less sensitive to cellular senescence than primary cells.

\section{Karyotype analysis of immortalized vole cells}

We analyzed the chromosomal karyotypes of the immortalized cells from both male and female voles (Table 3). Importantly, normal chromosome patterns were observed in about $30 \%$ of mitotic cells of the VMF-K4DT 

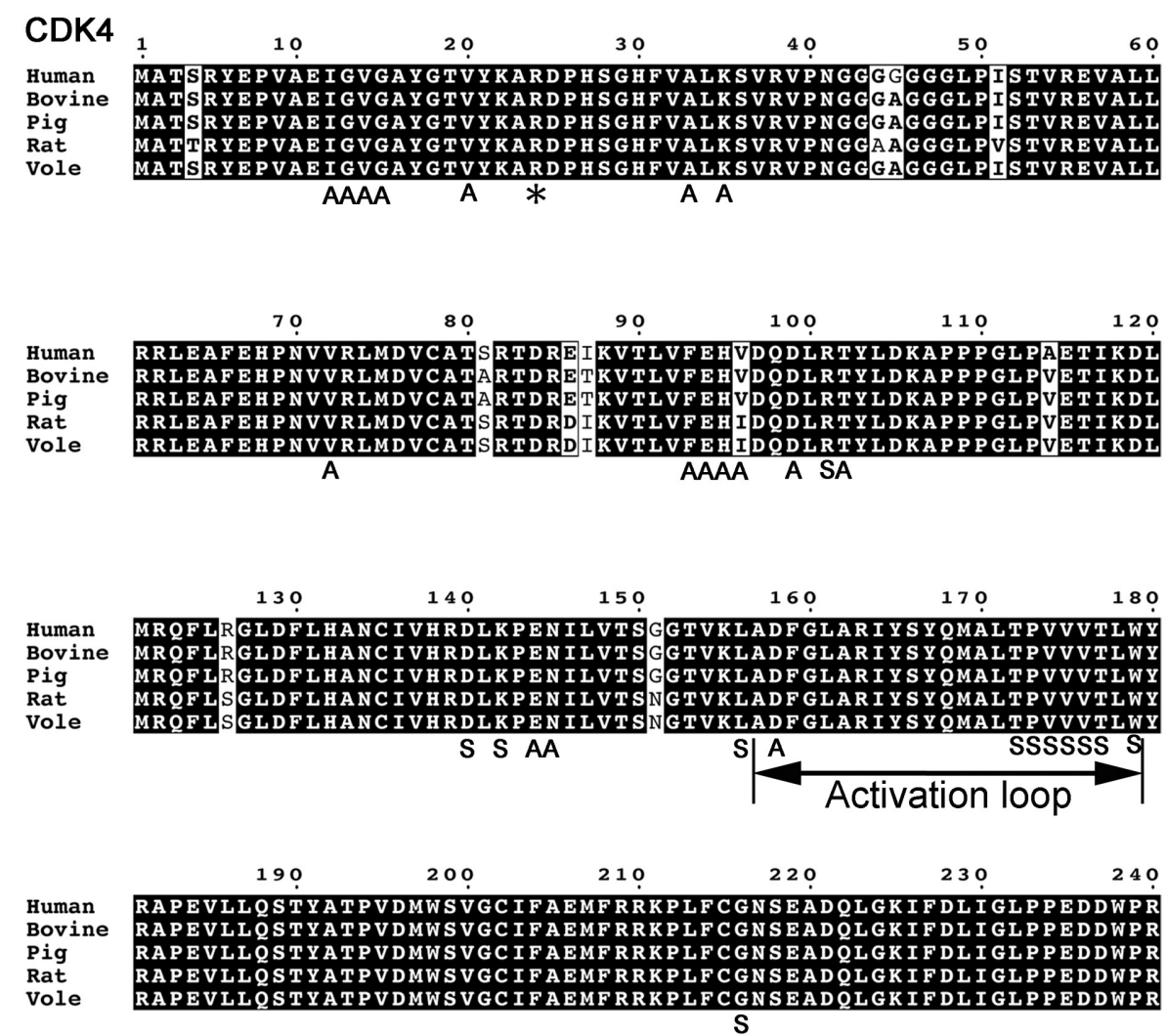

$\mathrm{S}$

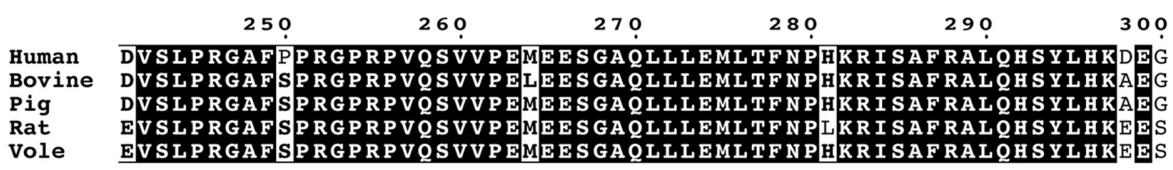

$\begin{array}{llll}\text { Human } & N & P E \\ \text { Bovine } & D & A E \\ \text { Pig } & N & P & E \\ \text { Rat } & D & P E \\ \text { Vole } & \text { D } & P E\end{array}$

Fig. 4. Alignment of human, bovine, pig, rat, and vole CDK4 amino acid sequences. Amino acid sequences were obtained from NCBI Gene (http://www.ncbi.nlm.nih.gov/gene), UCSC Genome Bioinformatics (https://genome.ucsc.edu), and Ensemble (http://asia.ensembl.org/index.html? redirect=no). Homology among the five species was analyzed by ESPript 3.0 (http://espript.ibcp.fr/ESPript/ESPript/). Core protein for binding p16 is indicated by an asterisk. In this figure, the corresponding positions of the ATP binding motif (A) and substrate binding motif (S) are shown.

No. 2 cell line (Fig. 6A), and chromosomal tetraploidization was observed in the remaining $70 \%$ of cells (Fig. 6B). Surprisingly, VMF-K4DT No. 1 cells, derived from a male vole, contained XXY sex chromosomes. In VMFK4DT No. 1 cells, a chromosomal deletion was also observed in chromosome number 22 (Fig. 6C). This deletion in chromosome 22 (deletion of both parental chro- mosomes or deletion of only one parental chromosome) was observed in all analyzed cells. In addition, mar (marker chromosome) 1, which did not match the banding pattern of any other chromosome, was observed in VMF-K4DT No. 1 cells (Fig. 6C). This nonhomologous chromosome may have arisen from genomic instability. 

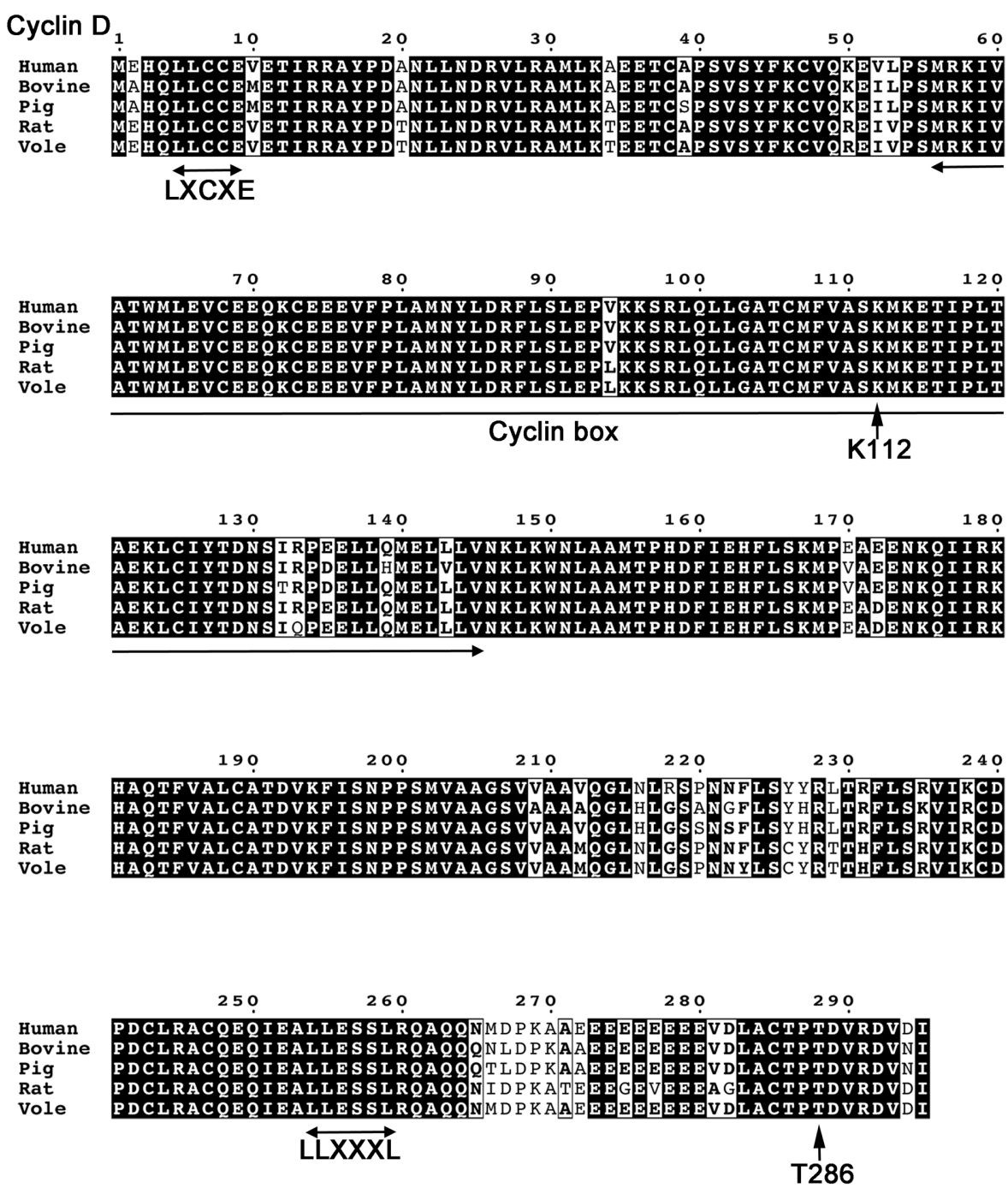

Fig. 5. Alignments of human, bovine, pig, rat, and vole cyclin D amino acid sequences. The corresponding positions for LXCXE, important for binding with RB, the cyclin kinase box, and LLXXXL, which is important for binding with steroid receptor co-activators, are shown.

Table 1. Results of cell cycle analysis of vole primary cells and immortalized cells

\begin{tabular}{lcccr}
\hline & G0/G1 & S & G2/M & \multicolumn{1}{c}{ Debris } \\
\hline Vole primary & $65.9 \pm 2.5$ & $4.6 \pm 0.4$ & $23.0 \pm 1.4$ & $19.3 \pm 0.7$ \\
VMF-K4DT No. 1 & $75.7 \pm 1.1$ & $4.4 \pm 0.3$ & $16.3 \pm 0.9$ & $8.3 \pm 0.5$ \\
\hline
\end{tabular}

Each value is shown as the mean $(n=4) \pm$ standard deviation $(\mathrm{SD})$.

Table 2. Transgene homology between the human and vole

\begin{tabular}{lccc}
\hline & CDK & Cyclin D1 & TERT \\
\hline Homology (between the human and vole) & $96 \%$ & $95 \%$ & $61 \%$ \\
\hline
\end{tabular}


Table 3. Karyotype analysis of the two immortalized cell lines

\begin{tabular}{cllllllllc}
\hline \multirow{2}{*}{ Cell line } & Gender & \multicolumn{5}{c}{ Chromosome number } & \multirow{2}{*}{$\begin{array}{c}\text { Total cell } \\
\text { number }\end{array}$} & $\begin{array}{c}\text { Normal chromosome } \\
\text { number ratio }\end{array}$ \\
\cline { 3 - 6 } & & 54 & 55 & 107 & 108 & 109 & & \\
\hline VMF-K4DT No. 1 & Male & 46 & 4 & & & & 50 & $92 \%$ \\
VMF-K4DT No. 2 & Female & 16 & & 4 & 28 & 2 & 50 & $32 \%$ \\
\hline
\end{tabular}

A

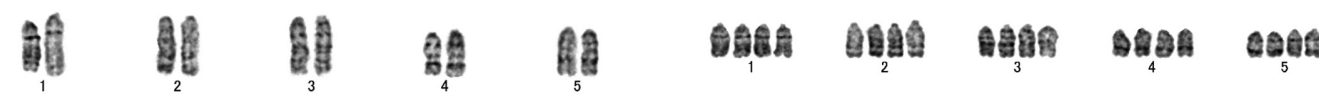

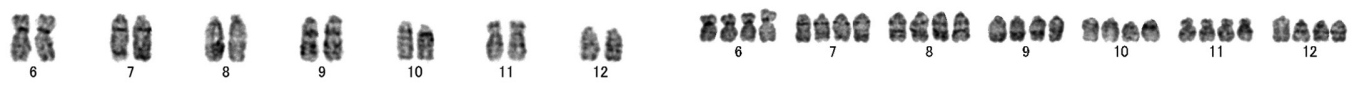

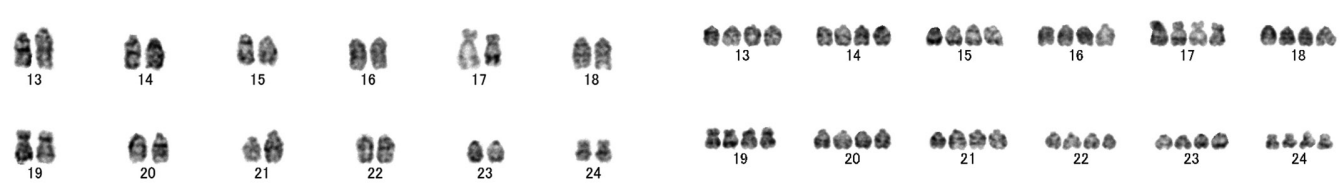

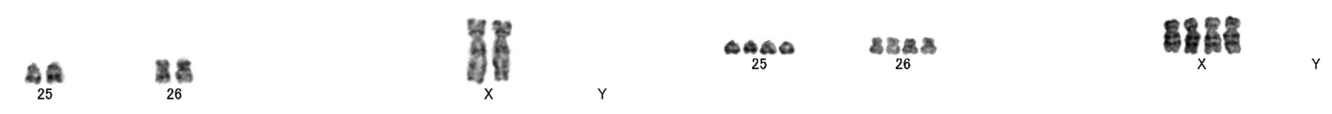

C

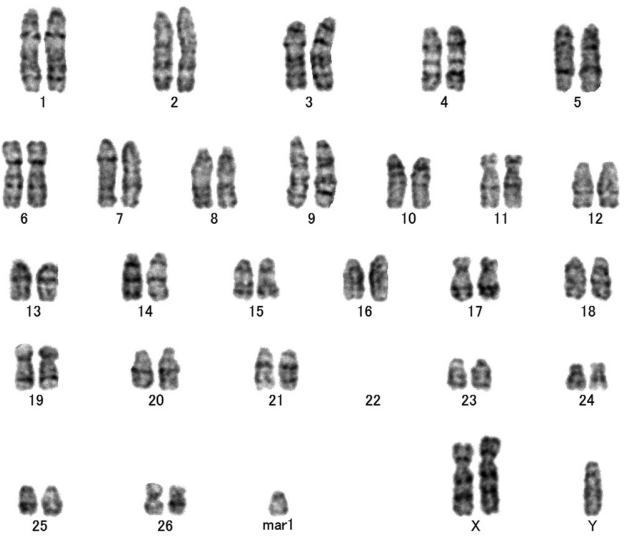

Fig. 6. Karyotype analysis of immortalized vole cells. (A) Normal chromosome in the immortalized vole cell (VMF-K4DT No. 2). (B) Chromosome tetraploidization observed in the immortalized vole cell (VMF-K4DT No.2). (C) Chromosome abnormality observed in the male-derived immortalized vole cell (VMF-K4DT No. 1). This cell has an XXY sex chromosome combination and is missing a portion of chromosome 22.

\section{Discussion}

In this study, we successfully immortalized prairie vole-derived fibroblasts by the expression of mutant CDK4 (CDK4R24C), cyclin D, and TERT. To the best of our knowledge, this is the first manuscript describing the establishment of immortalized vole-derived cells.

Our data showed that the immortalized vole-derived fibroblasts did not undergo cellular senescence. Accord- ing to our previous study, p16 protein expression increases during cellular senescence [18]. The accumulation of p16 protein results in the inactivation of endogenous CDK4 and the cyclin D complex, resulting in reduced cell proliferation. However, because of the presence of a point mutation, CDK4R24C is neither bound to nor inactivated by the $\mathrm{p} 16$ protein. These cell cycle regulators are known to be highly conserved during evolution, allowing us to immortalize vole-derived 


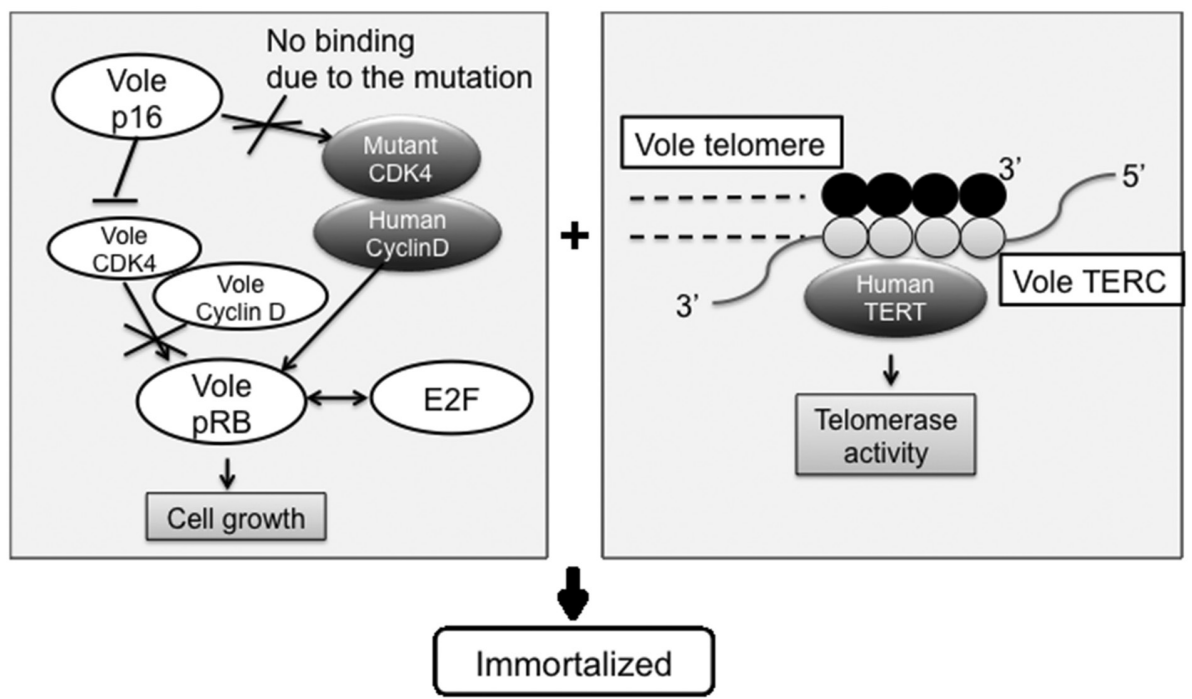

Fig. 7. Study summary. Immortalized cell system used in this study. In this study, mutant CDK4, cyclinD, and TERT were expressed.

cultured cells by inducing expression of human-derived CDK4 and cyclin D.

We used human-derived CDK4 and cyclin D in this study. These proteins exhibited high levels of homology with those of voles (CDK4, 96\%; cyclin D, 95\%; Table2). Alignment of the CDK4 amino acid sequences from multiple vertebrates is shown in Fig. 4. We included bovine and porcine amino acid data because we previously showed that cells from these two types of livestock are suitable for immortalization by expression of mutant CDK4, cyclin D, and TERT [5]. In CDK4, the 24th amino acid residue, which is important for binding to p16, is conserved in all species. Furthermore, the activation loop of CDK4 is conserved in all species. In cyclin $\mathrm{D}$, the central region known as the cyclin box is well conserved in all species (Fig. 5). Moreover, K112 (which is thought to act as a CDK4 binding site) and T286 (which affects ubiquitin-dependent proteolysis of cyclin D1) are conserved in all species (Fig. 5) $[3,7,11]$. The LLXXXL motif, which is important for binding with steroid receptor co-activators, is also conserved in all species. The LXCXE sequence has been reported to play an important role in the binding of $\mathrm{pRB}[14,19]$ and is also conserved in all species. The LXCXE region found in humans is identical to that found in voles (Fig. 5) [14, 19]. These results suggested that both livestock-derived (previously published) and vole-derived cells can also be immortalized due to the high conservation among multiple species.

Telomerase activity was detected in VMF-K4DT cells.
This result indicated that our introduced human-derived TERT formed a complex with vole-derived endogenous TERC and successfully functioned as a telomerase. Interestingly, we did not detect telomerase activity in volederived primary fibroblasts. As previously reported, telomerase activity is detectable, even in terminally differentiated cells collected from mouse tissue [15]. Although voles are also rodents, regulation of endogenous telomerase activity in voles may differ from that in mice.

In this study, we demonstrated for the first time that expression of mutant CDK4, cyclin D, and TERT was an effective method for immortalizing vole fibroblasts (Fig. 7). Our previous studies showed that expression of mutant CDK4, cyclin D, and TERT could be used to established immortalized bovine, porcine, and monkey cells $[5,12]$. In these previous studies, we did not observe any chromosomal abnormalities in swine, cattle, or monkeys. However, we detected chromosomal abnormalities in around $70 \%$ of the established vole cells. Although the reason for the chromosomal abnormalities in voles remains unclear, voles may simply be more sensitive to genomic instability. We observed tetraploid abnormalities only in the cells derived from a female vole (i.e., VMF-K4DT No. 2 cells). Because of the limited number of established cell lines, the relationship between gender and genomic abnormalities is not clear. We are now using immortalized cells derived from other rodents, such as mice, to confirm this hypothesis.

In this study, we successfully established prairie vole- 
derived cells. Prairie voles may be a useful animal model for a variety of research areas. For example, in recent studies, the bank vole has been shown to exhibit efficient transmission of human prions [2]. Our cells are able to expand continuously, and they can be shared with scientists worldwide. Thus, prairie vole-derived cells may be a useful bioresource for screening in various fields of study.

\section{Acknowledgments}

We would like to thank Dr. Emiko Isogai (Graduate School of Agricultural Science, Tohoku University) for assistance with the cell cycle analysis. This study was supported by JSPS KAKENHI grant number 25640117.

\section{References}

1. Carter, C.S. and Getz, L.L. 1993. Monogamy and the prairie vole. Sci. Am. 268: 100-106. [Medline] [CrossRef]

2. Chianini, F., Cosseddu, G.M., Steele, P., Hamilton, S., Hawthorn, J., Síso, S., Pang, Y., Finlayson, J., Eaton, S.L., Reid, H.W., Dagleish, M.P., Di Bari, M.A., D’Agostino, C., Agrimi, U., Terry, L., and Nonno, R. 2015. Correlation between infectivity and disease associated prion protein in the nervous system and selected edible tissues of naturally affected scrapie sheep. PLoS ONE 10: e122785. [Medline] [CrossRef]

3. Diehl, J.A., Zindy, F., and Sherr, C.J. 1997. Inhibition of cyclin D1 phosphorylation on threonine-286 prevents its rapid degradation via the ubiquitin-proteasome pathway. Genes Dev. 11: 957-972. [Medline] [CrossRef]

4. Dimri, G.P., Lee, X., Basile, G., Acosta, M., Scott, G., Roskelley, C., Medrano, E.E., Linskens, M., Rubelj, I., PereiraSmith, O., Peacocke, M., and Campisi, J. 1995. A biomarker that identifies senescent human cells in culture and in aging skin in vivo. Proc. Natl. Acad. Sci. USA 92: 9363-9367. [Medline] [CrossRef]

5. Donai, K., Kiyono, T., Eitsuka, T., Guo, Y., Kuroda, K., Sone, H., Isogai, E., and Fukuda, T. 2014. Bovine and porcine fibroblasts can be immortalized with intact karyotype by the expression of mutant cyclin dependent kinase 4, cyclin D, and telomerase. J. Biotechnol. 176: 50-57. [Medline] [CrossRef]

6. Donaldson, Z.R. and Young, L.J. 2008. Oxytocin, vasopressin, and the neurogenetics of sociality. Science 322: 900904. [Medline] [CrossRef]

7. Feng, Q., Sekula, D., Müller, R., Freemantle, S.J., and Dmitrovsky, E. 2007. Uncovering residues that regulate cyclin D1 proteasomal degradation. Oncogene 26: 5098-5106. [Medline] [CrossRef]

8. Fukuda, T., Katayama, M., Yoshizawa, T., Eitsuka, T., Mizukami, H., Nakagawa, K., Ito, H., Komagata, H., Song, S., Roh, S., Hoshino, Y., Sato, E., Hanada, H., Nishimori, K., Miyazawa, T., and Uchida, T. 2012. Efficient establishment of pig embryonic fibroblast cell lines with conditional ex- pression of the simian vacuolating virus 40 large $\mathrm{T}$ fragment. Biosci. Biotechnol. Biochem. 76: 1372-1377. [Medline] [CrossRef]

9. Haff, R.F., and Swim, H.E. 1956. Serial propagation of 3 strains of rabbit fibroblasts; their susceptibility to infection with vaccinia virus. Proc. Soc. Exp. Biol. Med. 93: 200-204. [Medline] [CrossRef]

10. Hayflick, L. 1965. The Limited in Vitro Lifetime of Human Diploid Cell Strains. Exp. Cell Res. 37: 614-636. [Medline] [CrossRef]

11. Kobayashi, H., Stewart, E., Poon, R., Adamczewski, J.P., Gannon, J., and Hunt, T. 1992. Identification of the domains in cyclin A required for binding to, and activation of, $\mathrm{p} 34 \mathrm{cdc} 2$ and $\mathrm{p} 32 \mathrm{cdk} 2$ protein kinase subunits. Mol. Biol. Cell 3: 1279-1294. [Medline] [CrossRef]

12. Kuroda, K., Kiyono, T., Eitsuka, T., Isogai, H., Takahashi, K., Donai, K., Isogai, E., and Fukuda, T. 2015. Establishment of cell lines derived from the genus Macaca through controlled expression of cell cycle regulators. J. Cell. Biochem. 116: 205-211. [Medline] [CrossRef]

13. Miyoshi, H. 2004. Gene delivery to hematopoietic stem cells using lentiviral vectors. Methods Mol. Biol. 246: 429-438. [Medline]

14. Mulligan, G. and Jacks, T. 1998. The retinoblastoma gene family: cousins with overlapping interests. Trends Genet. 14: 223-229. [Medline] [CrossRef]

15. Prowse, K.R. and Greider, C.W. 1995. Developmental and tissue-specific regulation of mouse telomerase and telomere length. Proc. Natl. Acad. Sci. USA 92: 4818-4822. [Medline] [CrossRef]

16. Qin, X.Y., Fukuda, T., Yang, L., Zaha, H., Akanuma, H., Zeng, Q., Yoshinaga, J., and Sone, H. 2012. Effects of bisphenol A exposure on the proliferation and senescence of normal human mammary epithelial cells. Cancer Biol. Ther. 13: 296-306. [Medline] [CrossRef]

17. Sasaki, R., Narisawa-Saito, M., Yugawa, T., Fujita, M., Tashiro, H., Katabuchi, H., and Kiyono, T. 2009. Oncogenic transformation of human ovarian surface epithelial cells with defined cellular oncogenes. Carcinogenesis 30: 423-431. [Medline] [CrossRef]

18. Shiomi, K., Kiyono, T., Okamura, K., Uezumi, M., Goto, Y., Yasumoto, S., Shimizu, S., and Hashimoto, N. 2011. CDK4 and cyclin D1 allow human myogenic cells to recapture growth property without compromising differentiation potential. Gene Ther. 18: 857-866. [Medline] [CrossRef]

19. Taya, Y. 1997. RB kinases and RB-binding proteins: new points of view. Trends Biochem. Sci. 22: 14-17. [Medline] [CrossRef]

20. Tsao, S.W., Wang, X., Liu, Y., Cheung, Y.C., Feng, H., Zheng, Z., Wong, N., Yuen, P.W., Lo, A.K., Wong, Y.C., and Huang, D.P. 2002. Establishment of two immortalized nasopharyngeal epithelial cell lines using SV40 large T and HPV16E6/ E7 viral oncogenes. Biochim. Biophys. Acta 1590: 150-158. [Medline] [CrossRef]

21. Young, L.J., Nilsen, R., Waymire, K.G., MacGregor, G.R., and Insel, T.R. 1999. Increased affiliative response to vasopressin in mice expressing the V1a receptor from a monogamous vole. Nature 400: 766-768. [Medline] [CrossRef] 